\title{
Nutrition support in cancer patients: a brief review and suggestion for standard indications criteria Gerson Peltz
}

Address: Associate Professor, The University of Texas at Brownsville, Biological Sciences Department, 80 Fort Brown Brownsville, TX 78520 U.S.A

E-mail: gpeltz@utb.edu

Published: 30 September 2002

Received: 16 August 2002

Nutrition Journal 2002, I:I

Accepted: 30 September 2002

This article is available from: http://www.nutritionj.com/content/I/I/I

(C) 2002 Peltz; licensee BioMed Central Ltd. This article is published in Open Access: verbatim copying and redistribution of this article are permitted in all media for any purpose, provided this notice is preserved along with the article's original URL.

Keywords: nutrition support, cancer, quality of life

\begin{abstract}
The indications of nutrition support in cancer patients have been subject to controversy. Most studies address the effects of the method in increasing the survival or the tumor response rate. Few studies have focused on the effects in improving quality of life.

After a brief review, we described the results of a study, which evaluated the effects of proteincaloric supplementation on the quality of life parameters in a group of head and neck cancer patients submitted to radiotherapy. The results support the suggestion of creating standard criteria to indicate nutrition support in cancer patients.
\end{abstract}

Based on our findings, nutrition support should be indicated for cancer patients considering the potential effects to improve the quality of life.

\section{Background}

Several studies have been published to evaluate the indications of nutritional support in cancer. The majority of these studies are review articles and meta-analysis. A consensus has been sought from the data analyses in order to establish a standard for nutritional support in cancer. However, the term "nutritional support" has been applied almost exclusively for the use of total parenteral nutrition, instead of the entire modalities of nutrition intervention, including nutritional supplementation and enteral nutrition. Moreover, several methodological errors have been shown, which has produced certain controversy in the data interpretation. Despite the inconsistencies observed, the general indications of nutrition support in cancer have resulted in guidelines and recommendations published in the medical literature. Three reviews were recently published addressing this issue.
In the first [1], the authors have reviewed 28 prospective randomized and controlled clinical trials evaluating the use of parenteral nutrition in cancer patients. The authors have concluded that parenteral nutrition can be used in the pre-operative period in patients having cancer of the gastrointestinal tract and it is beneficial in the reduction of major surgical complications and surgical mortality. On the other hand, no statistically significant benefit could be demonstrated in survival rate, treatment tolerance, treatment toxicity, and treatment response from patients submitted to chemotherapy and radiation therapy. Indeed, there was an increase of the risk of infection in patients submitted to chemotherapy and receiving parenteral nutrition, underscoring the importance of demonstrating significant benefits in randomized trials before parenteral nutrition is used routinely in these patients. Twenty-two of these studies were reported in full paper form and 6 as abstracts only. The quality of reporting was poor, with 
only $46 \%$ of applicable items being reported between the surgery, chemotherapy, or radiotherapy trials. Based on the adopted criteria for data evaluating, the review has showed the following characteristics:

1) $73 \%$ of the studies showed eligibility criteria, $41 \%$ indicated the source of patients, and $77 \%$ gave the patient characteristics;

2) The treatment plan was often clearly defined in $86 \%$ of the trials, but only $36 \%$ gave details of the therapy actually received;

3) Although the study objectives were described adequately in $77 \%$ of the studies, only $14 \%$ clearly described the method of randomization;

4) No study described the use of a second party review or even an internal review of the data. Information on censored data was reported only in $38 \%$ of the studies;

5) Basic statistical methods were reported in $64 \%$ of the studies, but an in-depth description was reported in only $27 \%$ of the studies;

6) Prognostic factors were used in analyzing the study results in $36 \%$, but this usually involved a subgroup analysis only, and

7) The statistical power was discussed only in 1 of the 22 trials.

In addition, the authors stated that the review:

a) Does not rule out a possible positive effect of parenteral nutrition in cancer patients submitted to chemotherapy and radiotherapy;

b) Has identified a small sample size in individual studies and the inability to group the data related to the end points, resulting in a high probability of not detecting a therapeutic effect, and

c) Has showed that patients severely malnourished were excluded from several studies, therefore reducing significantly the chances to identify the potential effect of parenteral nutrition in those patients with significant weight loss or unable to maintain an adequate nutritional intake.

The second review is a Position Paper from the American College of Physicians [2]. A total of 12 randomized and controlled trials were evaluated to estimate the effects of parenteral nutrition on survival and tumor response rates in patients undergoing chemotherapy. The end points were efficacy and effectiveness of the method and safety, which result in recommendations known as CEAP Statement (Clinical Efficacy Assessment Project Statement). The CEAP evaluates and informs College members and others about the safety and efficacy of diagnostic and therapeutic modalities and medical practices. The CEAP statements represent a synthesis of the literature and expert opinion and are intended to reflect the current state-ofthe-art knowledge concerning technology and medical practice. The results of the pool analysis have shown that parenteral nutrition did not improve the tumor response rate and patients receiving parenteral nutrition were $81 \%$ as likely to survive as control patients. For short-term survival (3 months), the best estimate of the odds ratio was 0.74, (with 95\% confidence limits ranging from 0.42 to 1.3). The effect of parenteral nutrition on chemotherapy toxicity also was assessed. In the studies reviewed, the use of parenteral nutrition was associated with four times the increased risk of significant infection. The pooled odds ratio was 4.1 , with $95 \%$ confidence limits of 2.4 to 6.9 . Although the recognition of considerable heterogeneity of patients, neoplasms, and circumstances of parenteral nutrition administration in the reviewed trials, and the reduced global number of patients, the formal statements and recommendations were:

- The routine use of parenteral nutrition for patients undergoing chemotherapy should be strongly discouraged;

- As most of patients in the studies reviewed were not severely malnourished, the net effect of parenteral nutrition in these patients is unknown, and

- Clinical trials with subgroups of patients or modifications of parenteral nutrition can only be justified if such research provides valid arguments that the effect of parenteral nutrition support will be different than that observed to date.

The third review is the American Society for Parenteral and Enteral Nutrition (ASPEN)'s Guidelines for the Use of Parenteral and Enteral Nutrition in Adult and Pediatric Patients [3]. It is recommending the following practice guidelines:

- Enteral tube feeding and parenteral nutrition support may benefit some severely malnourished cancer patients or those in whom gastrointestinal or other toxicities are anticipated to preclude adequate oral nutritional intake for more than 1 week. Patients who are candidates for nutrition intervention under these circumstances should receive nutrition support, if possible, in conjunction with the initiation of oncologic therapy; 
Table I: Quality of life scores for each group

\begin{tabular}{|c|c|c|c|c|c|c|}
\hline \multirow[t]{2}{*}{ EORTC QLQ C-30 } & \multicolumn{3}{|l|}{ SPCe } & \multicolumn{3}{|c|}{ Controlf $^{f}$} \\
\hline & $\mathrm{Al}$ & $\mathrm{A} 2$ & A3 & $\mathrm{Al}$ & $\mathrm{A} 2$ & A3 \\
\hline Physical functioning & 54 & 60 & 82 & 56 & 54 & 51 \\
\hline Emotional functioning & 62 & 70 & 78 & 64 & 64 & 70 \\
\hline Fatigue & 56 & 58 & 54 & 58 & 64 & 72 \\
\hline Pain & 42 & 40 & 40 & 46 & 50 & 58 \\
\hline Appetite loss & 34 & 32 & 32 & 32 & 40 & 52 \\
\hline Global health status & 62 & 66 & 76 & 60 & 54 & 52 \\
\hline
\end{tabular}

\footnotetext{
aThe questionnaire is composed of both multi-item scale and single item measures that range in score from 0 to 100. bA high scale score represents a higher response level for physical functioning and emotional functioning.

cA high score for the global health status represents high QoL.

dA high score for fatigue, pain, and appetite loss represents a high level of symptoms.

eStatistically significant results in PCS group pre-treatment period vs. post-treatment period $(p<0.05)$.

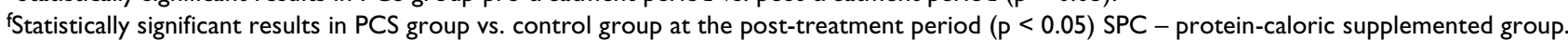

Al : one week before radiotherapy.

A2 : 30 days after the beginning of radiotherapy.

A3 : 60 days after the beginning of radiotherapy.
}

- Specialized nutrition support is not routinely indicated for well-nourished or mildly malnourished patients undergoing surgery, chemotherapy, or radiation treatment and in whom adequate oral intake is anticipated, and

- TPN is unlikely to benefit patients with advanced cancer whose malignancy is documented as unresponsive to chemotherapy or radiation therapy.

For the studies analyzed in the reviews, the conclusion drawn was correct: TPN was not beneficial. However, many people misinterpreted the conclusions without understanding the nuances of the issues being considered. This led, in turn, to erroneous assumptions about appropriate nutrition management in cancer patients in general [4].

More recently, studies have been published suggesting the need to consider quality of life (QoL) as end point in cancer trials [5-8]. The studies evaluating efficacy and effectiveness are taking into account the tumor response rate, survival, and QoL assessment as well. The use of QoL questionnaires has significantly modified the methodological procedure in cancer clinical trials. Therefore, the indication criteria for nutrition support must also incorporate the QoL assessment.

Klein and Koretz [9] brought first the idea that QoL assessment should be present in clinical trials involving nutrition support in cancer patients. The authors reviewed 70 prospective randomized controlled trials of nutrition support in more than 4000 cancer patients and they found the same results as previously described. However, the authors highlighted the following shortcomings associated with the reviewed studies:

- The statistical power of most studies was poor because of small sample size and most trials were unlikely to demonstrate a therapeutic effect of parenteral nutrition even if parenteral nutrition was efficacious;

- The patient population enrolled in the studies was heterogeneous consisting of patients with different tumor types and stages of disease;

- The composition of nutrients, timing of nutrition intervention, and duration of nutrition therapy differed among studies, making it difficult to pool data and to evaluate the efficacy of the method;

- Specific cancer therapy varied among studies, therefore increasing the possibility of confounding the conclusions made after grouping studies together;

- The patient populations were not stratified in accordance with their nutritional status, and in many trials the patients were not limited to those who might benefit most from nutrition support, that is, those with severe weight loss; 
Table 2: Classification of nutritional status:

\begin{tabular}{cccc}
\hline & Nourished & At risk of malnutrition & Malnourished \\
\hline Stage I & la & Ib & Ic \\
Stage II & Ila & Ilb & Ilc \\
Stage III & IIla & IIIb & IIlc \\
Stage IV & IVa & IVb & IVc \\
\hline
\end{tabular}

- The quality of reporting most studies was poor;

- Quality of life and other additional endpoints were rarely evaluated.

In which extension nutrition support can improve QoL in cancer patients, even knowing that the traditional end points will not be substantially modified? The use of QoL questionnaires developed to best evaluate QoL in cancer patients has addressed this issue [10-16].

\section{Methods}

In a study published elsewhere [17], our group prospectively compared the QoL effects of nutrition support in one hundred-forty-six patients with head and neck cancer submitted to radiotherapy receiving protein-caloric supplementation in routine basis (PCS). Briefly, protein-caloric supplementation in a routine basis (PCS) was administered to 72 patients (PCS group). The supplementation consisted of a polymeric formula containing 794 cal and $6,72 \mathrm{~g} \mathrm{~N}_{2}$, which was daily supplemented with the regular supervised diet beginning seven days before radiotherapy continuing seven days after the last day of treatment. The control group $(n=74)$ received nutritional counseling and supervised diet with no nutritional supplementation. The QoL was evaluated using the European Organization for Research and Treatment of Cancer Quality of Life Questionnaire (EORTC QLQ C-30) in three instances: one week before radiotherapy (A1), 30 days (A2) and 60 days (A3) after the beginning of therapy. This tool is composed of both multi-item scale and single item measures that range in score from 0 to 100 . A high scale score represents a higher response level. A high score for a functional scale (physical functioning, and emotional functioning) represents a high/healthy level of functioning, high score for the global health status represents high QoL, and high score for a symptom scale (fatigue, pain, and appetite loss) represents a high level of symptomatology/problems [18]. Statistical analysis was performed using the $t$-test for paired samples and the Student $t$-test.

\section{Results and Discussion}

The PCS group had high scores in physical functioning and emotional functioning and low scores in the scale of appetite loss. In contrast, the control group showed a persistent low score in physical functioning and a high score in the symptom scale such as fatigue, pain and appetite loss. In the analysis of global health status, the PCS group showed a high score and the control group showed a low score. Statistically significant results are shown in Table 1. The results have showed that protein-caloric supplementation in a routine basis improved the QoL in head and neck cancer patients submitted to radiotherapy and should be considered as a useful supportive practice. Moreover, these findings support the idea that studies related to the effects of nutrition support in cancer should incorporate the QoL assessment as a primary endpoint.

\section{Conclusions}

Based on the results of QoL evaluation in cancer patients, the indications of nutrition support should be expanded and reviewed. The criteria to prescribe nutrition support have to take into account the following aspects:

1) Related to the effectiveness of the anti-neoplastic therapy:

- Stage I - Curative, that is, the anti-neoplastic treatment is potentially curative

- Stage II - Advanced stage disease, that is, the tumor response rate is low due to the aggressive behavior of the cancer or the staging of the disease

- Stage III - No therapy to be offered, that is, there is no effective therapeutic strategy and usually the treatment is supportive (for instance, anti-hemorrhagic radiotherapy)

- Stage IV - Terminal, that is, the end stage disease leaving the patient severely limited in his functions

2) Related to the nutritional status

- a - Nourished 
- b - At risk of malnutrition

- c - Malnourished

The nutritional assessment should be performed using the Global Subjective Assessment or through the following criteria:

- Nourished: absence of weight loss of more than $5 \%$ of the usual body weight in the last 30 days

- At risk of malnutrition: weight loss of $5-10 \%$ of body weight within the last 30 days

- Malnourished: weight loss of more than $10 \%$ within the last 30 days

The patients should be further classified as shown in Table 2.

Nutrition support should be indicated based on the stage of the oncologic process and the nutritional status. The standard criteria for indication of nutrition support would follow the scheme below:

Ia, IIa, IIIa, IVa $\rightarrow$ follow up (periodical nutritional assessment)

Ib, IIb, IIIb, IVb $\rightarrow$ protein-caloric supplementation and/ or appetite stimulants

Ic, IIc, IIIc $\rightarrow$ nutritional therapy (enteral nutrition, total parenteral nutrition, and peripheral parenteral nutrition)

IVc $\rightarrow$ enteral fluids / venous fluids (hydration)

Patients submitted to bone marrow transplantation and those undergoing gastrointestinal surgery are not targeted by these criteria. In both groups, nutrition support is indicated in a routine basis.

Standard criteria to indicate nutrition support in cancer patients should be implemented and QoL assessment is an unconditional tool to determine the target population. Therefore, nutrition support teams should consider QoL benefits as an endpoint in further studies of the role of nutrition support in cancer.

\section{Competing interests}

None declared.

\section{References}

I. Klein S, Simes J, Blackburn GL: Total parenteral nutrition in cancer clinical trials. Cancer 1986, 58:1378-I38|

2. American College of Physicians: Parenteral nutrition in patients receiving cancer chemotherapy. Ann Intern Med 1989, I I 0:734736
3. American Society for Parenteral and Enteral Nutrition Board of Directors: Guidelines for the use of parenteral and enteral nutrition in adult and pediatric patients. JPEN 1993, I 7(Suppl): ISA-52SA

4. Bloch AS: Feeding the cancer patient: Where have we come from, where are we going? NCP 1994, 9:87-89

5. Browman GP, Levine MN, Hodson I, Sathya J, Russel R, Skingley P, Cripps $C$, Eapen L, Girard A: The head and neck radiotherapy questionnaire: a morbidity/quality-of-life instrument for clinical trials of radiation therapy in locally advanced head and neck cancer. J Clin Oncol 1993, I I:863-872

6. Tchekmedyian NS, Cella DF: Treatment of cancer anorexia with megestrol acetate: impact on quality of life. Oncology 1990, 4:185-92

7. Blazeby JM, Williams MH, Alderson D, Fardon JR: Observer variation in assessment of quality-of-life in patients with oesophageal cancer. Br J Surg 1995, 82:1200-1203

8. Olschewski M, Schulgen G, Schumacher M, Altman DG: Quality-oflife assessment in clinical cancer research. Br J Cancer 1994, 70: $1-5$

9. Klein S, Koretz RL: Nutrition support in patients with cancer: what do the data really show? NCP 1994, 9:91-100

10. Bergner M: The Sickness Impact Profile (SIP). In: Assessment of quality-of-life in clinical trials of cardiovascular therapies (Edited by: Wenger NP, Mattson ME, Furberg (D) New York, Le Jacq 1984, I52-I 59

II. Kaplan RM, Anderson JP: The General Health Policy Model: an integrated approach. In: Quality-of-life assessments in clinical trials (Edited by: Spilker B) New York, Raven 1990, I31-149

12. Stewart AL, Ware JE: Measuring functioning and well-being: The Medical Outcomes Study approach. Durham, NC, Duke University Press 1992

13. Nelson EC, Landgraf JM, Hays RD, Wasson JH, Kirk JW: The functional status of patients: how can it be measured in physicians' offices? Med Care 1990, 28: I III-II26

14. Chambers LWLW: The McMaster Health Index Questionnaire: an update. In: Quality-of-life: assessment and application (Edited by: Walker SR, Rosser RM) London, Ciba Foundation 1998, I I3-I3 I

15. McSweeny AJ, Creer T: Health-related quality-of-life assessment in medical care. Dis Mon 1995, 4 I: | -7|

16. Aaronson NK, Ahmedzai S, Bergman B, Bullinger M, Cull A, Duez NJ, Filiberti A, Flechtner H, Fleishman SB, de Haes JC, et al: The European Organization for Research and Treatment of Cancer QLQ-C30: a quality-of-life instrument for use in international clinical trials in oncology. J Natl Cancer Inst 1993, 85:365-376

17. Peltz G, Pinho NB, Najman HL, Aguiar MMM: Nutrition therapy and quality of life in cancer patients submitted to radiotherapy. Rev Bras Nutr Clin 1997, 12:S127

18. EORTC Study Group on Quality of Life: The European Organization for Research and Treatment of Cancer QLQ-C30: Scoring Manual Appendix Brussels 1997, 5:43 